\title{
EI Uso de una Aplicación Móvil en la Enseñanza de la Lectura
}

\author{
Martha Jiménez-García y Ma. de los Ángeles Martínez-Ortega \\ (1) Instituto Politécnico Nacional, Unidad Profesional Interdisciplinaria de Ingeniería y Ciencias Sociales y \\ Administrativas (UPIICSA), Sección de Estudios de Posgrado e Investigación. Av. Té 950, Iztacalco, \\ Granjas México, 08400 Ciudad de México (e-mail: majimenez@ipn.mx; marthajimenezga@gmail.com) \\ (2) Instituto Politécnico Nacional, Escuela Superior de Ingeniería y Arquitectura, Unidad Tecamachalco. Av. \\ Fuente de los Leones 28, San José de Los Leones 3era Sección, 53760 Naucalpan de Juárez, México. \\ (e-mail: mmartinezo@ipn.mx; mamigcar@hotmail.com)
}

Recibido Jun. 17, 2016; Aceptado Ago. 29, 2016; Versión final Oct. 3, 2016, Publicado Feb. 2017

\begin{abstract}
Resumen
El objetivo de este estudio fue analizar el uso de una aplicación móvil que facilita el proceso del aprendizaje de la lectura en la población adulta que presenta analfabetismo. El estudio se enmarca en un concepto global promovido por la UNESCO que recomienda que se ofrezca una educación de calidad para todos y que se impulse haciendo uso de la tecnología actual. Asimismo, el gobierno de México muestra una preocupación por disminuir el analfabetismo y el rezago educativo. Como instrumento de estudio se usó una aplicación móvil en Tablets bajo la plataforma Android y se aplicó a 143 personas adultas. En los resultados se encontró un alto impacto en el aprendizaje, pues el tiempo promedio de aprendizaje se redujo. Se concluye que el uso de la aplicación móvil en adultos, tiene un impacto positivo en el proceso de Enseñanza-Aprendizaje, ya que resultó una herramienta satisfactoria y muy fácil de usar.
\end{abstract}

Palabras clave: analfabetismo; aplicación móvil; educación; tecnología; Android

\section{Use of a Mobile Application in Teaching Reading}

\begin{abstract}
The aim of the study presented in this paper was to analyze the use of a mobile application, to facilitate the learning process of reading of illiterate adult population. The study fits in a more global concept promoted by UNESCO that recommends the provision of quality education for all people, using present technology. Additionally, the Mexican government is interested in reducing illiteracy and educational backwardness. The instrument used was a mobile application in Tablets operating under Android that was applied to 143 participants. The application was designed under an intuitive environment with audio instructions, selecting colors and images. The results found a high impact on teaching-learning, as the average time was reduced and users were highly satisfied.
\end{abstract}

Keywords: illiteracy; mobile application; education; technology; Android 


\section{INTRODUCCIÓN}

La Organización para la Educación, la Ciencia y la Cultura de las Naciones Unidas UNESCO (2009) estableció en su declaración "Educación para Todos" doce compromisos para garantizar educación de calidad a todos los habitantes del mundo, entre los cuales se destaca la creación de espacios seguros que propicien una educación integral que fomente la equidad, la armonía y un aprendizaje significativo, así como el aprovechamiento de los nuevos recursos que las Tecnologías de la Información y Comunicación (TIC) promueven y que favorezcan una educación incluyente. En México el Plan Nacional de Desarrollo 20132018 establece que se requiere fortalecer el capital humano para que genere una educación de calidad y se vea reflejada en una sociedad más justa y más próspera. Se requiere de un Sistema Educativo Mexicano vanguardista, acorde a las demandas del mundo globalizado. Una sociedad sin educación está predeterminada a fracasar en muchos aspectos, al no tener las herramientas necesarias que la modernidad demanda. Uno de los objetivos del Plan Nacional de Desarrollo es garantizar la inclusión y la equidad en el Sistema Educativo y para ello indica que se establezcan alianzas con instituciones de educación superior y organizaciones sociales, con el fin de disminuir el analfabetismo y el rezago educativo (Presidencia de la República, 2013).

El Programa Sectorial de Educación 2015-2018 manifiesta que existe otro problema que es urgente atender y es ese rezago educativo, que involucra a la población analfabeta mayor de 15 años que no han concluido su educación primaria o secundaria y que está conformado por 32.3 millones de mexicanos. Para ir subsanando esta situación, se pretende que los modelos para la educación de los adultos deban ser flexibles y pertinentes para atender las demandas de dicha población. Las nuevas tecnologías pueden ser muy útiles para este propósito (Secretaría de Educación Pública, 2013). En México existen más de 5 millones de personas en situación de analfabetismo, 3.9 millones de personas hablan español (México) y 1.5 millones hablan lenguas indígenas. Respecto a la distribución geográfica de estas personas, el Instituto Nacional de Estadística y Geografía (INEGI) reporta que un $49.7 \%$ se encuentra en zonas urbanas, mientras que el $50.3 \%$ reside en alguna comunidad rural. Si tomamos en cuenta la edad de este grupo de personas, los datos del censo arrojan que el $64 \%$ se encuentra en edad productiva, es decir, las edades oscilan entre los 15 y los 64 años de edad; mientras que el 36\% restante tiene 65 o más años (INEGI, 2010).

El objetivo de disminuir el analfabetismo planteado en el Plan Nacional de Desarrollo, los compromisos de la UNESCO, relacionados con una educación de calidad y el aprovechamiento y contribución de las TIC en el alcance de los objetivos de la educación, los que potencian nuevos métodos y modelos pedagógicos, ratificado por los autores Criado y Moreno (2009), promueven este trabajo en busca de un aporte a la educación a través del uso de las TIC, de tal forma que se dé respuesta a las preguntas de investigación: ¿Existe relación de las TIC basadas en una aplicación móvil de la enseñanza de la lectura para el aprendizaje de la lectura? ¿El uso de una aplicación móvil en una Tablet facilita el aprendizaje de la lectura en el adulto? ¿Es fácil utilizar una Tablet por el adulto para aprender a leer? ¿El uso de una aplicación móvil motiva al adulto para aprender a leer?.

En esta investigación se presenta el resultado de un experimento mediante el empleo de la Aplicación móvil para el aprendizaje de la lectura, denominada Legendi, desarrollada para plataforma Android; bajo la hipótesis de que el uso de las TIC facilita el proceso de aprendizaje de la lectura y el desarrollo cognitivo en la población adulta analfabeta. La investigación es aplicada, transversal y experimental. El objetivo de este documento es analizar el uso de una aplicación móvil elaborada bajo la plataforma Android, para facilitar el proceso del aprendizaje de la lectura en la población adulta analfabeta.

El analfabetismo es un adjetivo que se usa para las personas que no saben leer ni escribir, también hace referencia a las personas que, aunque identifican letras, palabras y/o números no son capaces de utilizar estas capacidades de manera eficiente en situaciones habituales (Martínez, Trucco, \& Palma, 2014). El Instituto Nacional para la Educación de los Adultos (INEA) diseñó y presentó la Campaña Nacional de Alfabetización y Abatimiento del Rezago Educativo que contempla, entre otros objetivos, en seis años (2013-2018) reducir el índice de analfabetismo del 2010 pasando del 6,9\% a 3,5\% al finalizar este periodo (INEA, 2013). Asimismo el INEA informa que del 2014 a 2018 se pretende atender: a) 2.2 millones de jóvenes y adultos rezagados en el nivel primaria b) 3.1 millones sin secundaria y c) 2.2 analfabetos (INEA, 2014).

Las habilidades de alfabetización están relacionadas con el crecimiento económico a nivel nacional (Hanushek y Woessmann, 2012); es por eso necesario innovar en algunos aspectos. En este caso se presenta una aplicación móvil instalada en Tablets, dispositivo o computador portátil, caracterizado por su tamaño pequeño y por pantalla táctil que no requiere de mouse (ratón), la cual incluye aspectos de sensación y percepción, así como una selección de imágenes. 
La sensación es un proceso en el que los órganos de los sentidos convierten estímulos del mundo exterior en datos elementales o materia prima de la experiencia (Consuegra, 2010). Con respecto a la percepción Myers (2005) argumentó que a partir de la sensación los objetos se pueden percibir a través de los sentidos y la mente; lo cual hace que se genere información con los aspectos de la sensación al estar presente la percepción, al ser diferente en cada persona según el tipo de actividades que se han experimentado. Asimismo, el proceso del pensamiento permite seleccionar, organizar e interpretar, es decir, se transforma la sensación en percepción, al darle un significado y organización en la memoria (Goldstein, 2011). La percepción se realiza en zonas de rango perceptual (figura y fondo) en las que se fija la atención. En cuanto a figura es el ente que existe en un espacio exaltando su interrelación con otros elementos y el fondo, se considera aquello que no es figura. De manera formal, figura-fondo constituye la integración de una totalidad o Gestalt. Por otro lado, dependiendo del lugar donde se fije la atención pueden aparecer diferentes figuras de lo que es el fondo (Zamudio, 2014).

El color es una característica resaltante en la imagen, le da atracción y estimulación, resultando atrayente para el observador, no olvidando que los colores son impactantes de manera consciente o inconsciente para el individuo observante (Ocampo, 2012). González (2011) indicó que el sonido es la percepción audible ocasionada por vibraciones ordenadas y captadas por los órganos auditivos, las cuales son transmitidas al cerebro mediante ondas sonoras. El diseño de sonido es esencial en las producciones multimedias. En el caso de aplicaciones móviles proporciona un nivel distinto de comunicación al presentar aspectos visuales, de audio y de tacto.

Cebolla y Agustí (2012) sugieren usar las TIC como un modo de innovación y educación de calidad. Sala (2013) comenta que las TIC, deben de manejarse con un fundamento teórico y con un sentido claro de sus finalidades educativas, para que se fortalezca el proceso de la enseñanza y el aprendizaje. Investigadores como Shapley et al. (2011), Fragoso y Gonzalez (2013) manifiestan en sus aportaciones que existe una gran apertura en el uso de las TIC. Işık (2013) indicó que existe una relación entre la tecnología y la economía basada en el conocimiento como objetivo del crecimiento económico y de la productividad. Bataller Sala (2013) argumentó que es necesario el uso de las TIC en el proceso Enseñanza-Aprendizaje (E-A) para tener una educación de calidad. La tecnología móvil ha revolucionado muchos aspectos de la vida en el mundo en desarrollo, en la Escuela de Negocios de Londres Banks (2014) y en varios estudios recientes realizados por Farhadi, Ismail, y Fooladi (2012), y Saidi, Hassen, y Hammami (2014), Chew, llavarasan, y Levy, (2015) entre otros, han encontrado que el uso de dispositivos móviles tiene un impacto positivo en el crecimiento económico. Asimismo, las competencias digitales de habilidades en el uso de TIC son esenciales en el ámbito educativo. Los profesores deberían cambiar la enseñanza con un enfoque tecnológico para maximizar el tiempo de clase mediante un aprendizaje activo (linuma, 2016).

El proceso de aprendizaje de los individuos con dispositivos móviles es una actividad relacionada con las TIC (Brown, 2005); por lo cual es necesario incluir en la educación el uso de dispositivos móviles. Morales (2012) comentó que el uso de las TIC potencia los aprendizajes colaborativos. Fong y Holland (2011), así como Peeraer y Petegem (2011) y Krishnan et al. (2012) señalan que los profesores están mejor preparados para la enseñanza con el uso de las TIC, ya que estas van en aumento cada día. Nour (2013) y Méndez (2013) indican y recomiendan que se promuevan las TIC en la educación. A través del uso de las TIC se ha permitido incrementar nuevos métodos y modelos pedagógicos con el uso de tecnología en repositorios que permiten dar respuestas en tiempo real, realidad aumentada con el uso de sensores, o bien el uso de juegos en mesas interactivas. Lo cual facilita el uso de herramientas didácticas que propician formas de aprendizaje y comunicación innovadoras entre el docente-discente y entre sus pares, cambiando totalmente la concepción del proceso E-A.

\section{METODOLOGÍA}

La investigación realizada es de tipo cualitativo, fue exploratoria de tipo no probabilístico e intencional. Asimismo, se realizó un análisis longitudinal. La metodología se presenta en varias subsecciones, 1) participantes, 2) objeto de estudio y 3) aplicación del instrumento.

\section{Participantes}

Los participantes fueron seleccionados mediante la accesibilidad del investigador, de tal forma que reunieran los requisitos del objetivo de la investigación, es decir, adultos mayores de 15 años que presentaban analfabetismo. El estudio se realizó en diferentes zonas geográficas de México, la selección de la muestra en cuanto a las zonas se realizó de forma aleatoria en el estado de México, en los municipios de Tlalnepantla, Naucalpan, Atizapán de Zaragoza, Nicolás Romero, Nezahualcóyotl. Se realizó en estas localidades debido a que estos son algunos municipios en donde se tiene mayor cantidad de personas analfabetas. Asimismo, se realizó en el estado de Oaxaca en el municipio de Tepelmeme Villa de Morelos, siendo este municipio una comunidad en pobreza extrema en donde también se presenta un alto porcentaje 
de analfabetismo, además de que se pudo analizar el impacto de la tecnología del uso de un dispositivo móvil en una comunidad por personas que no habían usado dicha tecnología. Por lo que la muestra quedó conformada por 143 personas, de una población de 467,734 personas, dicha muestra fue superior al tamaño de la muestra esperado de 73personas.

\section{Objeto de estudio: aplicación móvil (Legendi)}

El objeto de estudio en este caso es la aplicación móvil (Legendi). Con el uso de esta aplicación se analiza el objetivo planteado al facilitar el proceso del aprendizaje de la lectura con el uso de la tecnología en personas con analfabetismo. Se utilizó esta aplicación en 6 sesiones a personas analfabetas para comprobar la hipótesis y confirmar que se propicia el aprendizaje de la lectura.

La aplicación fue diseñada tomando como guía el material didáctico utilizado por el Instituto Nacional para la Educación de los Adultos. Legendi es un prototipo de aplicación desarrollado para plataforma Android, con la finalidad de enseñar a las personas mayores de 15 años las reglas básicas de lectura del idioma español (México). La aplicación se encuentra diseñada bajo un entorno intuitivo, basado en instrucciones de audio, selección de colores e imágenes. Dicha aplicación facilita el aprendizaje de la lectura.

La aplicación móvil incluye ejercicios intuitivos y prácticos, incluyendo un apartado extra de ejercicios de comprensión de oraciones. La complejidad de los ejercicios es muy básica en los primeros capítulos, pero poco a poco se incrementa el nivel de dificultad para fomentar y desarrollar las destrezas y la memoria del educando al recordar lecciones pasadas y en un momento dado, inclusive motivar de manera autogestionada a la persona, para regresar a un capítulo previo, si resulta necesario repasar algunos ejercicios. Es necesario resaltar que el audio de las instrucciones se diseñó para que fueran breves y claras, esto con el fin de no distraer al educando del contenido base de los ejercicios. En las figuras 1 y 2 se muestran la pantalla de bienvenida y el menú principal respectivamente.
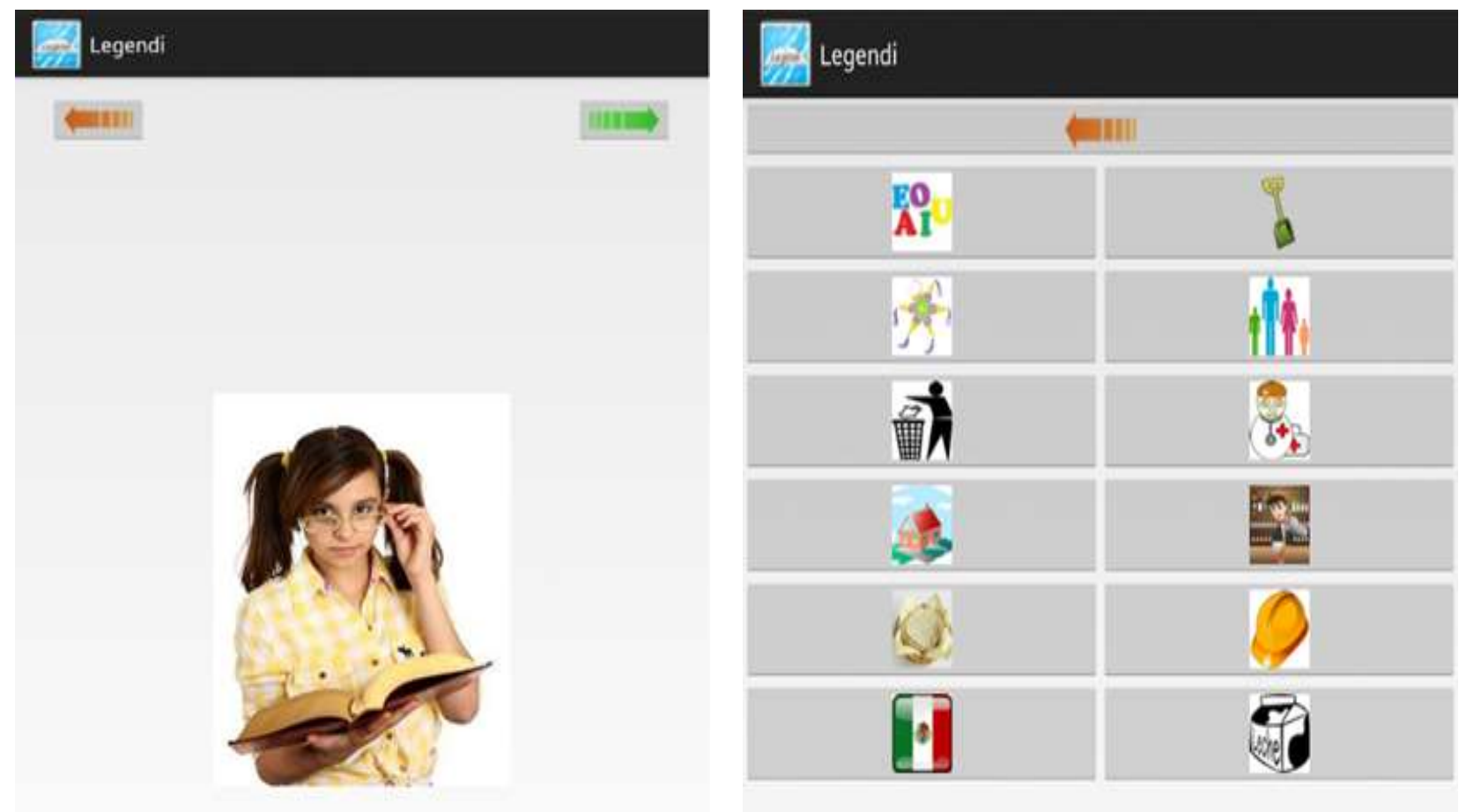

Fig. 1: Pantalla de bienvenida de Legendi

Fig. 2: Menú de Legendi.

En la figura 3 se presenta el primer acercamiento del educando con las vocales, con la finalidad de escuchar el sonido; posteriormente en la figura 4 se incluyen algunos ejercicios básicos para identificar palabras e imágenes que contienen la vocal estudiada. Asimismo, la figura 4 es un ejemplo de los tipos de ejercicios que se encuentran frecuentemente dentro de la aplicación. Como se observa, la base son las imágenes, las instrucciones vía audio indican que es necesario identificar el sonido del nombre de las imágenes, para saber si alguna de ellas cuenta con la vocal indicada. Es importante resaltar que se eligieron imágenes conocidas por la gran mayoría de las personas de las comunidades, objeto de estudio. 
Asimismo, se incluyó la parte de motivación, pues se tienen cuatro imágenes como opciones de respuesta, sin embargo, sólo una de éstas es la correcta. La respuesta acertada lanzará inmediatamente un mensaje de audio con la exclamación "¡Muy bien!”. Simultáneamente, a este mensaje en la pantalla se visualizará la imagen de una cara feliz, para posteriormente, desvanecerse junto con la pantalla del ejercicio, dando paso así a la siguiente lección o ejercicio según sea el caso. Tal como se muestra en la figura 5 , cuando el educando seleccione alguna de las tres opciones que son incorrectas, el mensaje de audio emitirá el mensaje "Esa no es, intenta de nuevo" a la vez que en la pantalla se muestra temporalmente una imagen de una cara triste, la cual se desvanece al cabo de un par de segundos para brindar al educando una oportunidad más de seleccionar la opción adecuada. El ejemplo de esto se observa en la figura 6.

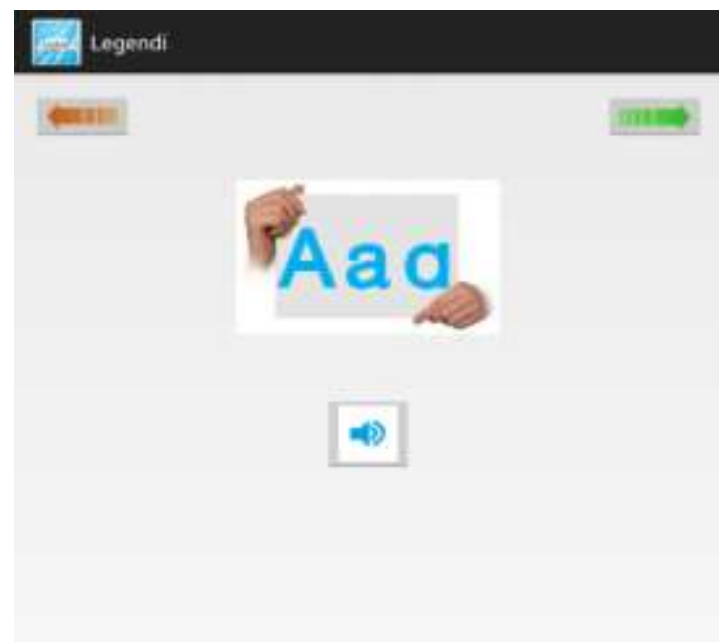

Fig. 3: Pantalla de familiarización de las vocales

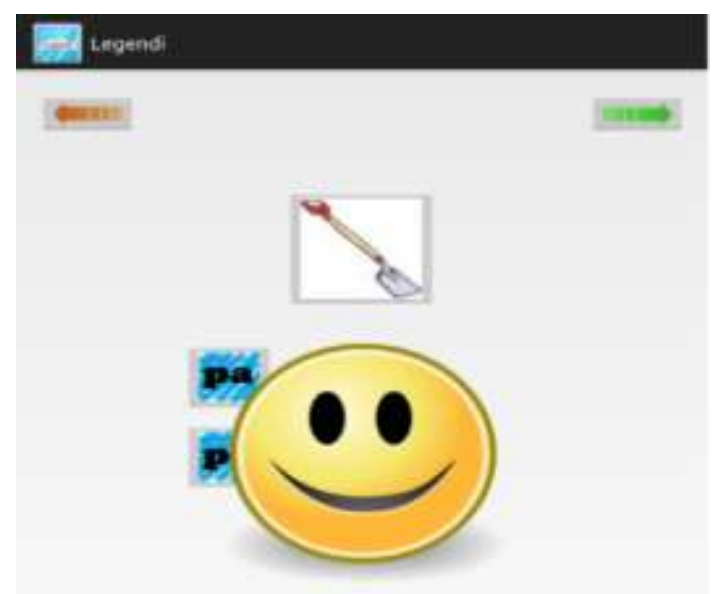

Fig. 5: Evento de Legendi a una respuesta acertada

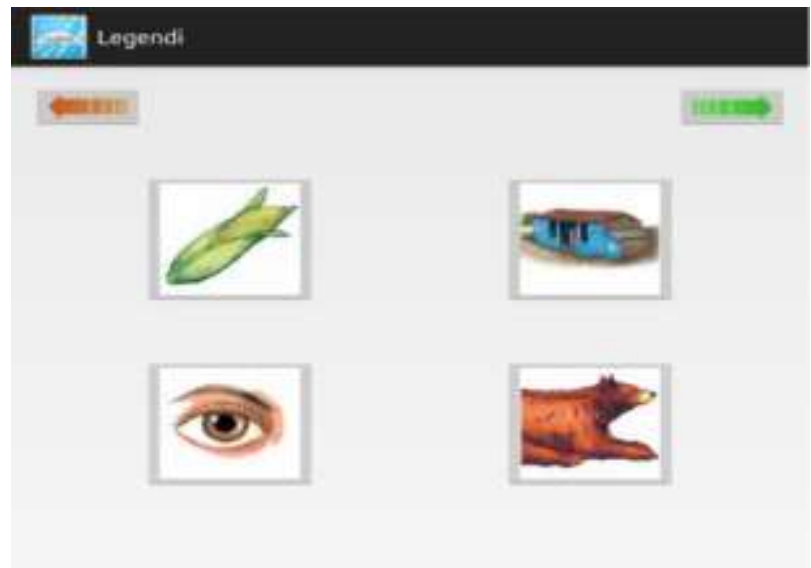

Fig. 4: Ejercicio de relación fonética-visual para vocales

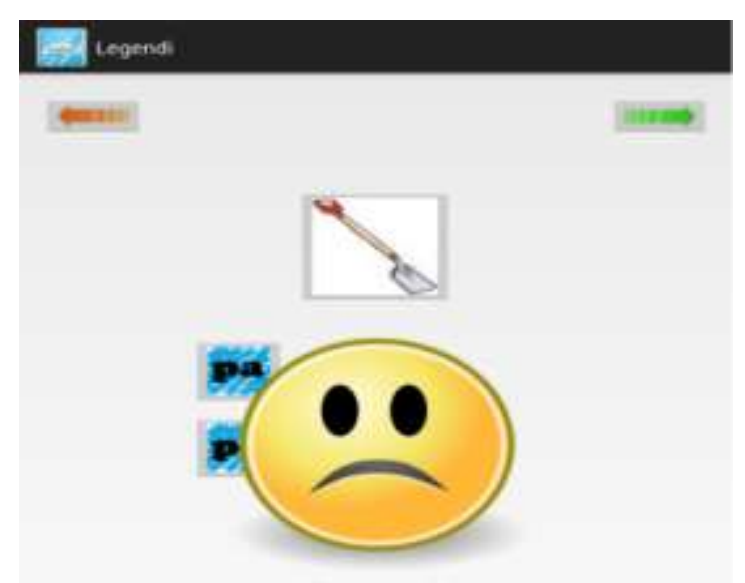

Fig. 6: Evento de Legendi a una respuesta equivocada

\section{Aplicación del instrumento}

El instrumento fue presentado a los participantes, quienes nunca habían usado un dispositivo móvil, pues algunos ni siquiera tienen teléfono celular. La aplicación fue instalada en Tablets. Se les explicó el funcionamiento de la aplicación para que adquirieran el conocimiento y habilidades en el aprendizaje de la lectura. Únicamente fueron necesarias 6 sesiones de dos horas. Después de 6 sesiones se les hizo un examen de seguimiento sobre el aprendizaje de la lectura. Posteriormente, se les dieron otras 2 sesiones y se les volvió a realizar un segundo examen sobre su aprendizaje de la lectura. Por último, se entrevistó a los participantes tomando como eje dos preguntas rectoras: 1. ¿Qué tan satisfecho se sintió, al usar la aplicación móvil en la Tablet? y 2. ¿Qué tan fácil fue usar la Tablet?, entre otras preguntas de tipo sociodemográfico como el número de integrantes de la familia, el ingreso mensual familiar. 


\section{RESULTADOS}

En la figura 7 se indica el resultado de las personas participantes en donde se aprecia la distribución de la muestra con relación a la edad, siendo la mínima de 41 años y la máxima de 90 años. De las personas participantes resultaron 63 mujeres y 80 hombres, como se muestra en la figura 8 . En la figura 9 se tiene el número de integrantes de la familia de la persona que utilizó la aplicación móvil para aprender a leer. Los integrantes oscilan entre 1 y 12 personas, aunque la mayor parte tiene una familia pequeña de 2 integrantes.

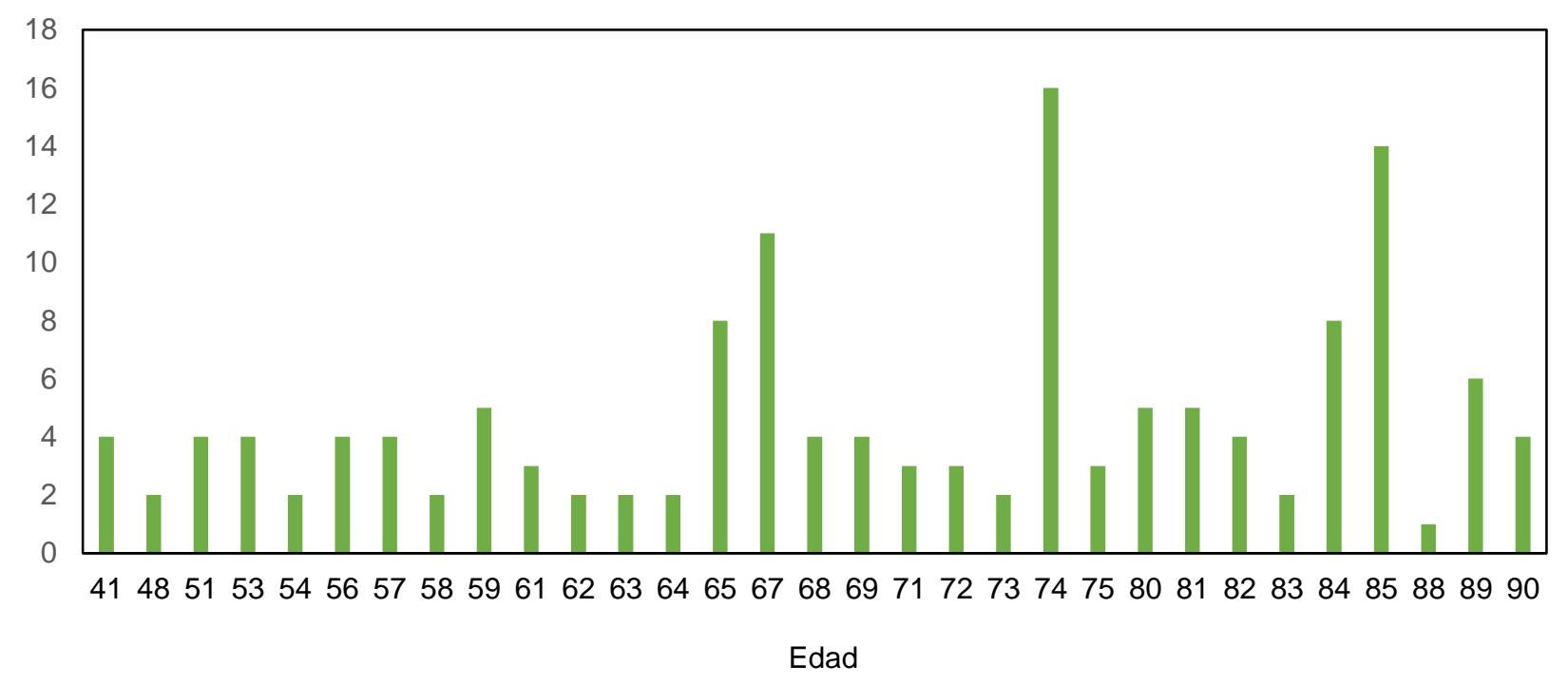

Fig. 7: Edad de los participantes en la aplicación del instrumento

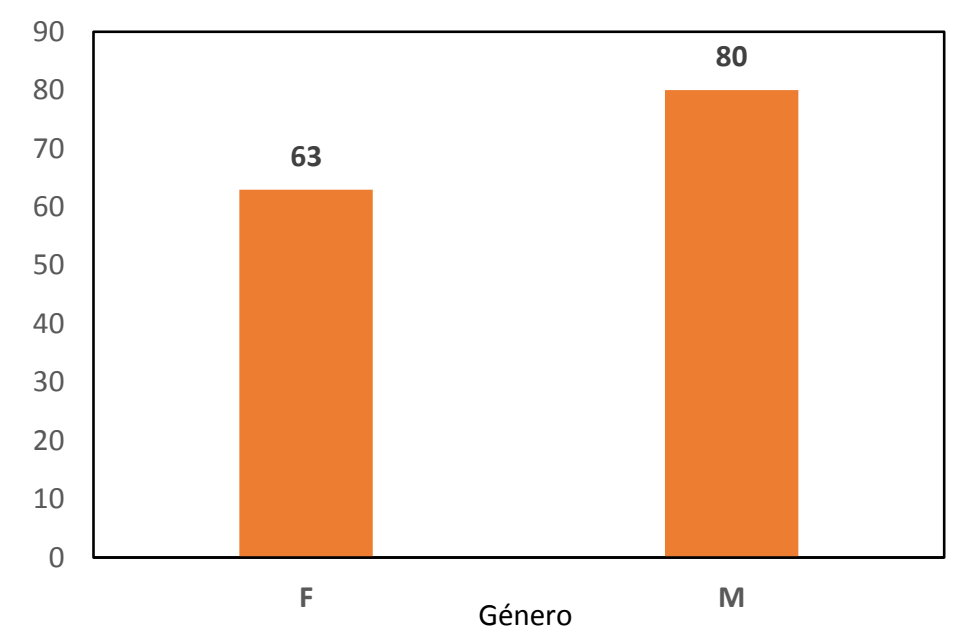

Fig. 8: Distribución de personas por género que utilizaron la aplicación móvil

En la figura 10 se indica el ingreso mensual familiar de los participantes en pesos mexicanos, el cual se indica desde 300 hasta 10,000 pesos y la mayor cantidad percibe solamente 1,200 pesos, también destacan los que perciben 6,000 pesos. En la figura 11 se muestra el resultado de la aplicación de los dos exámenes de evaluación sobre el aprendizaje de la lectura. El primer examen fue después de recibir 6 sesiones como ya se ha comentado anteriormente de 2 horas y el segundo examen fue sólo para confirmar la fluidez de la lectura, al darles 2 sesiones más de práctica, es decir 8 sesiones. Asimismo, se aprecia la disminución del tiempo del examen en minutos.

La tabla 1 indica que un alto grado de satisfacción en el uso de la aplicación, pues el $69 \%$ está satisfecho. Asimismo, en la tabla 2 se indica que usar la Tablet fue muy fácil, pues el52\% así lo expresó. 


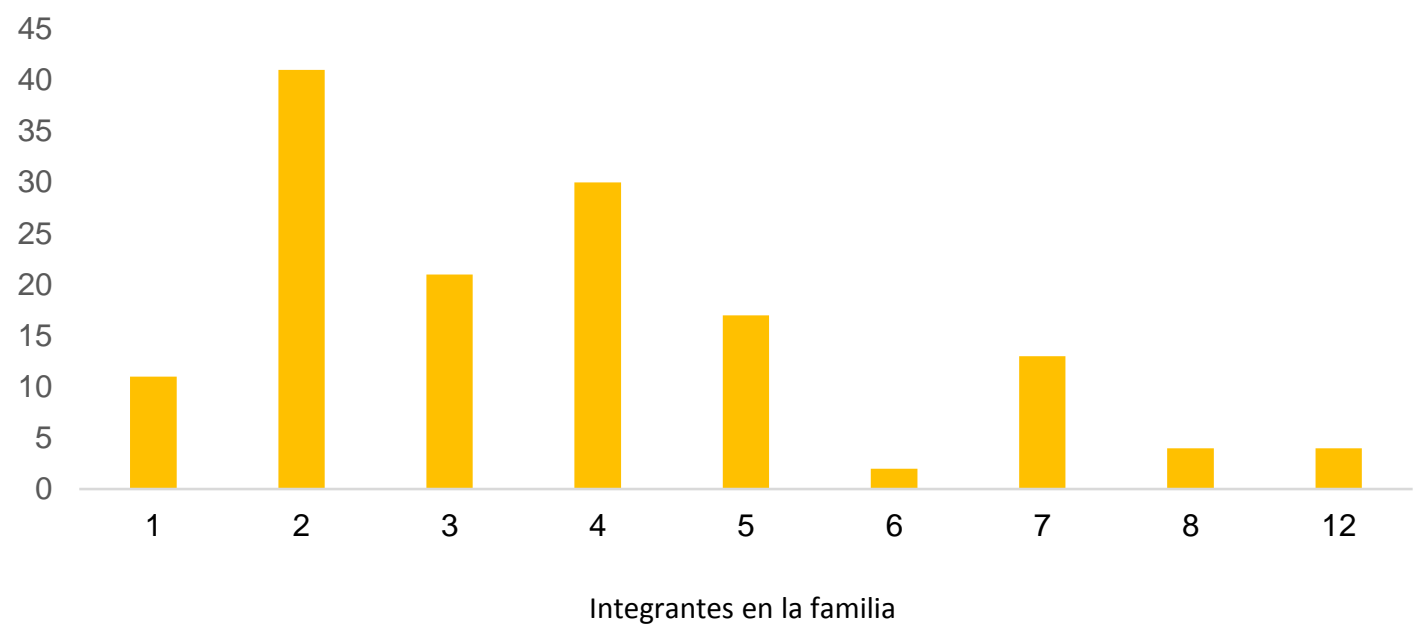

Fig. 9: Integrantes de la familia de las personas participantes en el uso de la aplicación móvil

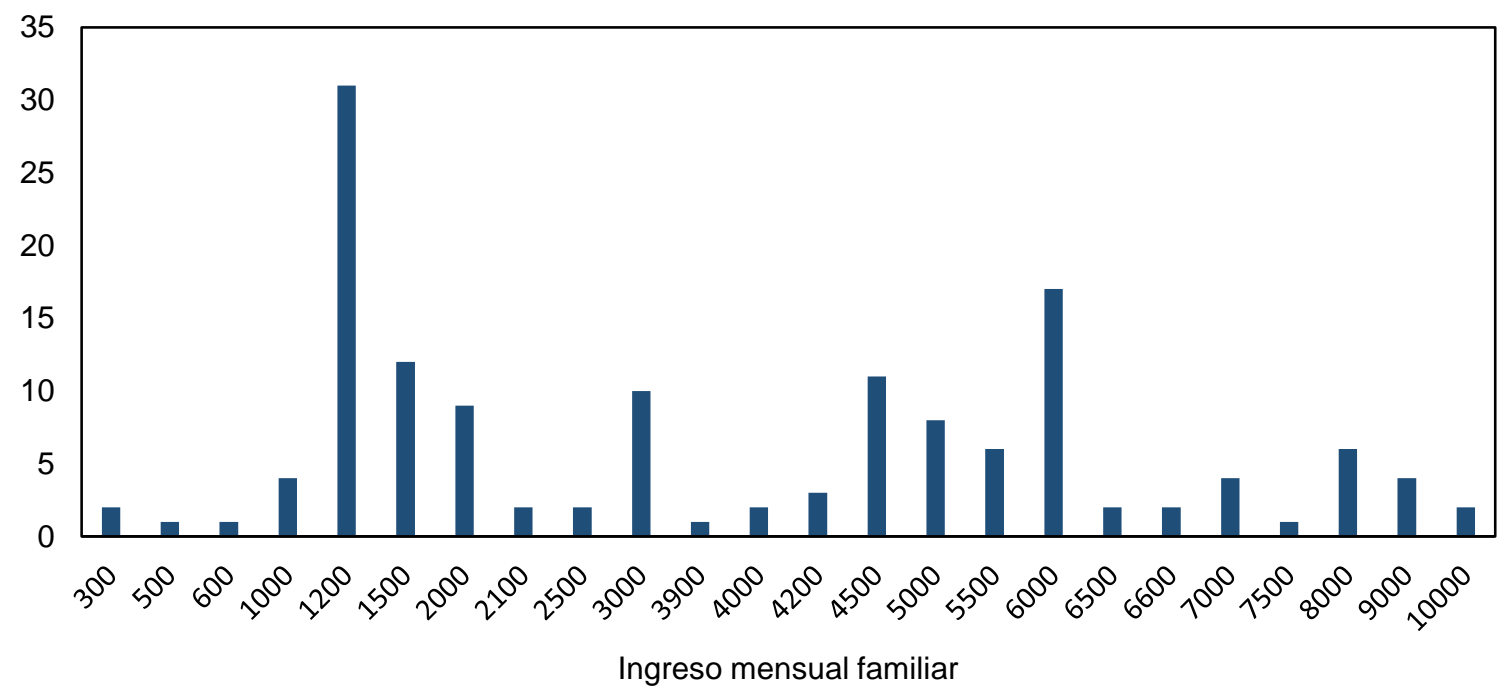

Fig. 10: Ingreso Mensual familiar de las personas participantes en el uso de la aplicación móvil

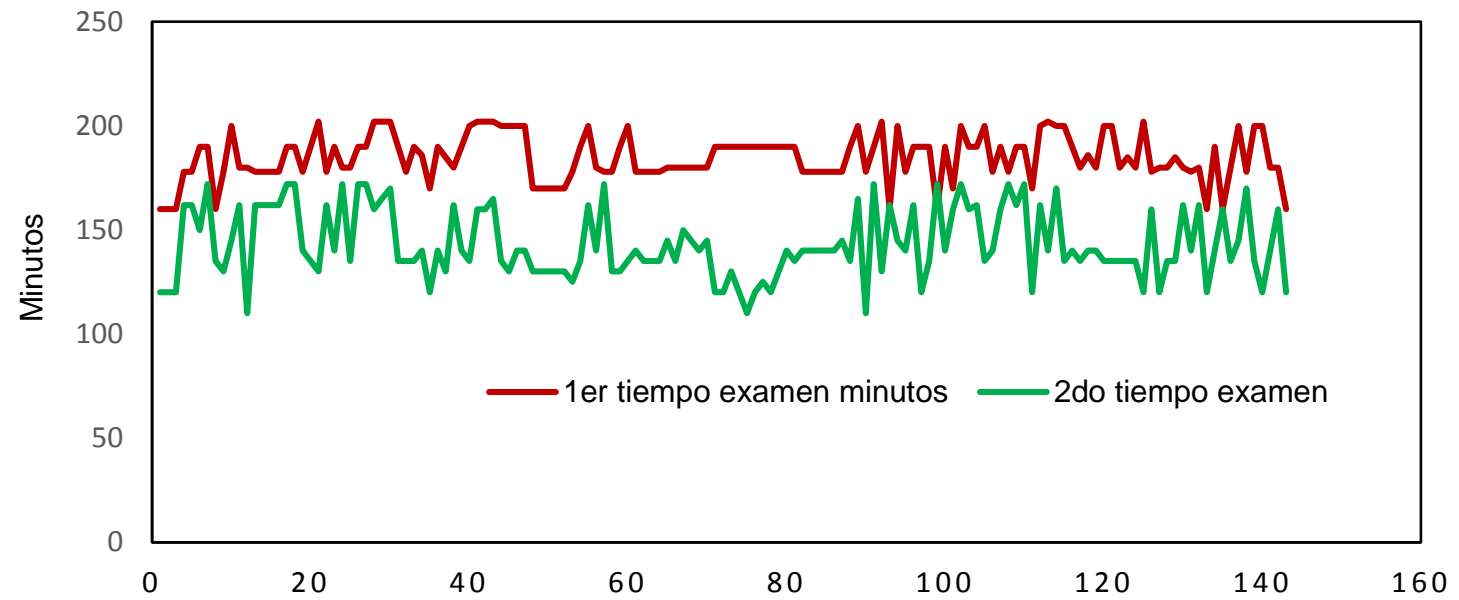

Fig. 11: Tiempo de respuesta en el uso de la aplicación móvil en la elaboración de 2 exámenes 
Tabla 1: ¿Qué tan satisfecho estuvo al usar la aplicación móvil

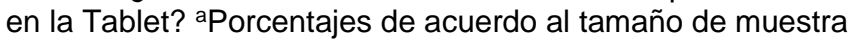

\begin{tabular}{lc} 
& $(\mathrm{n}=143)^{\mathrm{a}} \%$ \\
\hline Muy Satisfecho & 69 \\
Un poco Satisfecho & 23 \\
Ni satisfecho ni insatisfecho & 6 \\
Un poco insatisfecho & 2 \\
Muy insatisfecho & 0 \\
\hline
\end{tabular}

Tabla 2: ¿Qué tan fácil fue usar la Tablet?

aPorcentajes de acuerdo al tamaño de muestra

\begin{tabular}{lc}
\hline & $(\mathrm{n}=143)^{\mathrm{a}} \%$ \\
\hline Muy fácil & 52 \\
Fácil & 33 \\
Algo Difícil & 12 \\
Difícil & 3 \\
Muy difícil & 0 \\
\hline
\end{tabular}

\section{ANÁLISIS DE RESULTADOS}

Como resultado importante de este estudio se tiene el rápido aprendizaje de la lectura de los adultos al pasar de 10 sesiones (el INEA del municipio de Chalco reporto que el promedio del aprendizaje para aprender a leer de los adultos es de 10 sesiones de 2 horas) a 6 sesiones, con un ahorro de 4 sesiones de 2 horas. Asimismo, se perfeccionó esta habilidad en 2 sesiones, lo cual se puede apreciar en la figura 11. Por lo que se cumplió el objetivo planteado y se confirma la hipótesis definida. Pero además la condición anímica que provocó en ellos, al utilizar la aplicación móvil en una Tablet, recurso vanguardista que provoca una sensación de inclusión social, pues los participantes indicaron que estaban muy satisfechos con el uso de la aplicación en una Tablet, además fue muy fácil manejarla, lo cual hace que tengan pertenencia en la sociedad digital, pues ahora ya han usado una Tablet.

En la figura 7 se percibe un amplio rango de edades de los participantes desde 41 hasta 90 años, donde se tiene que la mayoría fueron hombres (figura 8); la mayor cantidad de participantes tiene 2 integrantes en la familia (figura 9). Además de que perciben un ingreso familiar muy bajo (figura 10). Sin embargo, pese a que algunos adultos ni siquiera tiene un teléfono celular de tipo smartphone y de que nunca habían usado una Tablet, presentaron interés en la aplicación móvil (Legendi) y un 69\% opinó a favor de la satisfacción que les proporcionó el uso de la tecnología mencionada (tabla 1), pues resultó ser muy fácil su uso (tabla 2 ).

Además de que el ingreso promedio mensual de los hogares en el país en 2014 se ubicó en \$13,247pesos, según la Encuesta Nacional de Ingresos y Gastos de los Hogares (INEGI,2014), lo que indica que las personas con problemas de analfabetismo presentan ingresos muy por debajo del ingreso promedio. Con lo cual se espera que, al tener la habilidad de lectura, se encuentren en igualdad de oportunidades para ser más competitivos y poder aumentar su ingreso familiar mensual.

\section{DISCUSIÓN}

Una aplicación móvil -sobre el aprendizaje de la lectura en tablets usada en personas adultas-, es algo muy esencial para la vida, ya que, al ser una habilidad importantísima, esta le permite al individuo, el desarrollo de abstracciones cada vez más finas que van conformando un pensamiento introspectivo cada vez más complejo. Este ascenso, permite al ser humano una mayor comunicación con sus semejantes y tener una mejor y positiva interacción con su entorno. Los educandos realizaron un total de 8 sesiones en un dispositivo móvil para aprender a leer, lo cual reflejó un aprendizaje rápido, y confirma lo indicado por Konrad et al. (2005), cuando mencionaron que el proceso de aprendizaje de la lectura en un adulto es más ágil en comparación con un niño, ya que su sistema atencional presenta una mayor madurez.

Es evidente la importancia del aprendizaje de la lectura, ya que es esencial para la vida (Romero, 2005). En los resultados de esta investigación se encontró que las personas con analfabetismo perciben ingresos económicos bajos. Una adecuada política educativa en TIC puede ser un instrumento para combatir la pobreza (Pedraza, López, \& Salcedo, 2012). Lo cual confirma que el uso de una aplicación móvil para aprender a leer, tiene un impacto positivo, además se estaría en igualdad de tener mejores 
oportunidades para la vida. Por lo que se recomienda facilitar el proceso de aprendizaje de la lectura con el uso de las TIC, ya que la educación es un pilar como fuente de crecimiento y bienestar económico. Es pertinente resaltar que el uso de las TIC en el proceso Enseñanza Aprendizaje, además de facilitarlo, genera en el usuario una condición de autonomía y pertenencia, ya que diluye barreras generacionales.

Esta investigación resaltó que el uso de las TIC en la alfabetización de personas adultas para el aprendizaje de la lectura, facilita los procesos de enseñanza aprendizaje y propicia una educación de calidad. El uso de esta aplicación, favorece y promueve principalmente dos propósitos universales: Una educación para todos con una condición incluyente y la reducción del rezago educativo. Además de apoyar a la generación de crecimiento económico en función de la tecnología, en este caso del uso de una aplicación móvil.

\section{CONCLUSIONES}

De los resultados mostrados, de su análisis y de su discusión, se pueden obtener las siguientes conclusiones sobre el uso de una aplicación móvil sobre el aprendizaje de la lectura: 1) facilita el aprendizaje de la lectura, 2) disminuye el tiempo de aprendizaje en la lectura 3) perfecciona la habilidad lectora, 4) es muy satisfactorio para los adultos usar dicha aplicación móvil, 5) es muy fácil utilizar esta aplicación. El uso de la aplicación móvil en la Tablet, evidenció su impacto positivo en el proceso de Enseñanza-Aprendizaje con adultos, ya que resultó una herramienta satisfactoria para el usuario y muy fácil de usar, por lo que generó una actitud autogestiva en su proceso de aprendizaje, que propicia una condición de pertenencia social.

\section{AGRADECIMIENTOS}

Agradecemos las facilidades que fueron otorgadas para la realización del presente trabajo al Instituto Politécnico Nacional - Unidad Profesional Interdisciplinaria de Ingeniería y Ciencias Sociales y Administrativas. Proyecto SIP 20161046 Las Habilidades Informáticas del Capital Humano como Fuente de Crecimiento Económico.

\section{REFERENCIAS}

Banks, K., Mobile learning in the last mile, doi:10.1007/s11125-013-9282-x, Prospects, 44(1), 5-11, (2014)

Bataller Sala, M., El uso didáctico de las tecnologías de la información y la comunicación (TIC) en la práctica docente de la licenciatura en pedagogía del sistema de universidad abierta ya distancia (SUAyED) de la UNAM, Amicus Curiae. Segunda Época, 2(1), (2013)

Brown, T.H., Beyond constructivism: Exploring future learning paradigms, Education Today, 2(2),1-11 (2005)

Cebolla, J. P. B. y Agustí, M. F., Estudios de caso sobre las percepciones de los estudiantes en la inclusión de las TIC en un Centro de Educación Secundaria, Profesorado. Revista de Currículum y Formación de Profesorado, 16(3), 285-305, (2012)

Consuegra, A., Diccionario de psicología. Bogotá: Ecoe Ediciones, (2010)

Criado, R. y Moreno, A. B., Una propuesta de enseñanza virtual y su aplicación a la asignatura "Matemáticas e Imaginación", RELADA-Revista Electrónica de ADA-Madrid, 1(2), 59-64, (2009)

Fong, R. W. \& Holland, T., A Study of Teachers' Beliefs and Practices of Using Information and Communication Technology (ICT) In Classrooms, doi: 10.1007/978-94-6091-427-0_11, Science Education in International Contexts, III, 143-158, (2011)

Fragoso, J. P. y Gonzalez, P., Didáctica de la educación en valores en la eso. Una propuesta utilizando las tecnologías, Pixel-Bit: Revista de medios y educación, (42), 195-208, (2013)

Goldstein, E., Sensación y Percepción, Cengage Learning, México, (2011)

González, F. B., El lenguaje musical en educación infantil, Cuadernos de Educación y Desarrollo, 3(27), (2011)

Hanushek, E. A. \& Woessmann, L., Do better schools lead to more growth? Cognitive skills, economic outcomes, and causation, doi: 10.1007/s10887-012-9081-x, J. Economic Growth, 17(4), 267-321, (2012)

linuma, M., Literacy Theories in the Knowledge Society, doi: 10.1007/978-981-10-0144-4_1 In Learning and Teaching with Technology in the Knowledge Society, (en línea), 1-13, Springer Singapore, (2016) 
INEA [Instituto Nacional para la Educación de los Adultos], Campaña Nacional de Alfabetización y Reducción del Rezago Educativo 2013.2018, INEA, (2013)

INEA [Instituto Nacional para la Educación de los Adultos], Participa en la campaña de alfabetización y abatimiento del rezago educativo. INEA, (en línea: http://www.inea.gob.mx/index.php/participacampana.html, acceso 13 Abril 2016), México (2014)

INEGI [Instituto Nacional de Estadistica y Geografía], Instituto Nacional de Estadistica y Geografía, México en cifras, Disponible en: http://www3.inegi.org.mx/sistemas/mexicocifras/, (2010)

INEGI [Instituto Nacional de Estadística, \& Informática] Encuesta nacional de ingresos y gastos de los hogares, México, Instituto Nacional de Estadística, Geografía e Informática, (2014)

Işık, C., The importance of creating a competitive advantage and investing in information technology for modern economies: an ARDL test approach from Turkey, doi: 10.1007/s13132-011-0075-2, Journal of the Knowledge Economy, 4(4), 387-405, (2013)

Konrad, K., Neufang, S., y otros seis autores. Development of attentional networks: an FMRI study with children and adults, Neuroimage, 28(2), 429-439, (2005)

Krishnan, S. N., Rahim, R. A., y otros 11 autores. ICT and Teachers' Attitude in English Language Teaching. Asian Social Science, 8(11), 8-12, (2012)

Martínez, R., Trucco, D. \& Palma, A., El analfabetismo funcional en América Latina y el Caribe: Panorama y principales desafíos de política, (2014)

Méndez Coca, D., Cambio motivacional realizado por las TIC en los alumnos de secundaria de física, Revista de Ciencias Humanas y Sociales, 136(70), 199-224, (2013)

Morales R.M., Actitudes e Intereses hacia las TIC en una Muestra de Estudiantes de Posgrado, Psicología educativa, 18(1) 53-63, (2012)

Myers, D., Psicología. España: Editorial Médica panamericana, (2005)

Nour, S. M., Education, Training and Skill Development Policies in Sudan: Macro-Micro Overview,doi: 10.1007/978-3-642-32811-4_9, In S. M. Nour, Technological Change and Skill Development in Sudán (en línea) (págs. 313-407), Berlín: Springer Berlín Heidelberg, (2013)

Ocampo, M. Á. G., El apoyo del diseño en la percepción en una Diapositiva, Investigación en Discapacidad, 1(1) 41-46, (2012)

Peeraer, a. J. \& Petegem, P. V., ICT in teacher education in an emerging developing country: Vietnam's baseline situation at the start of 'The Year of ICT', Computers \& Education, 56, 974-982, (2011)

Pedraza, L. F., López, D. A., \& Salcedo, O. J., Brecha digital por estatus socio-económico en la Localidad de Ciudad Bolívar de Bogotá (Colombia). Información tecnológica, 23(6), 63-72, (2012)

Presidencia de la República, Plan Nacional de Desarrollo 2013 2018. México: Gobierno de la República, (2013)

Romero, L., Aprendizaje de la Lecto-escritura, Fe y Alegría, Perú, (2005)

Sala, M. C. B., El Uso Didáctico de las Tecnologías de la Información y la Comunicación (TIC) en la Práctica Docente de la Licenciatura en Pedagogía del Sistema de Universidad Abierta y a Distancia (SUAyED) de la UNAM, Revista Amicus Curiae, Segunda Época, 2(1), (2013)

Secretaría de Educación Pública, Programa Sectorial de Educación 2013-2018, (2013)

Shapley, K., Sheehan, D., Maloney, C. y Caranikas-Walker, F., Effects of technology immersion on middle school students' learning opportunities and achievement, doi: 10.1080/00220671003767615, The Journal of Educational Research, 104(5), 299-315, (2011)

UNESCO, Organización de las Naciones Unidas para la Educación, la Ciencia y la Cultura, Conferencia Mundial sobre la Educación Superior/2009: la nueva dinámica de la educación superior y la investigación para el cambio social y el desarrollo, Unesco, París, (2009)

Zamudio, J. F. Á., Sueños y Fantasía: Propuesta de Categorías de Análisis en Terapia Gestalt Para Grupos de Adultos Mayores, Revista Electrónica de Psicología Iztacala, 17(1), 226, (2014) 